\title{
LONG-PERIOD VARIABLES AND CARBON STARS IN THE
}

\section{GALACTIC BULGE}

\author{
PATRICIA WHITELOCK \\ South African Astronomical Observatory, \\ P O Box 9, Observatory, Cape 7935, South Africa
}

October 12,1992

\begin{abstract}
The review covers the properties of red variables in globular clusters and the Galactic Bulge. Details are given of our current understanding of the Mira evolutionary phase. There is evidence that Miras in the LMC and the Bulge occupy different parts of the instability strip but obey the same PLC relation. The Bulge contains at least $2 \times 10^{4}$ Niras of which 100 or so have luminosities in excess of $\mathrm{M}_{\mathrm{bol}}=-5 \mathrm{mag}$. The Mira phase lasts more than $10^{5} \mathrm{yr}$. These objects originate from stars with a wide range of metallicity, but it is currently unclear if the most metal-rich stars reach the top of the AGB to become Miras. Preliminary data suggest that the distribution of the Miras along the minor axis of the Bulge is different from that of the late-M stars but similar to the $2.4 \mu \mathrm{m}$ luminosity.

Our knowledge of the Bulge carbon stars is briefly reviewed. It is suggested that, by analogy with the carbon-rich dwarfs, these stars are probably best understood as the products of binary evolution.
\end{abstract}

Key words: Galactic Bulge - Mira Variables - LPVs - IRAS sources - Carbon Stars - Metallicity Gradients

\section{Introduction}

Studies of variable stars have made a substantial contribution to our understanding of the Galactic Bulge, and show promise of doing the same for the bulges of other galaxies. Detailed investigations of the red variables, the typical flux distribution of which peaks longward of $1 \mu \mathrm{m}$, had to await the development of infrared techniques and surveys (see Glass's contribution to these proceedings). In particular, the wide variety of studies which followed the IRAS survey have made a major contribution to the field. This review describes our current understanding of low-mass red variables as derived from studies of globular clusters and the Galactic Bulge. Some emphasis is given to the Mira variables which, although they are the most luminous objects in the Bulge, are far from being fully understood. The review covers recent work on the period-luminosity-colour (PLC) relation which was discovered for Miras in the LMC but is proving crucial to our understanding of Miras in the Bulge. It also appears that the distribution of Miras in the Bulge follows the bulk luminosity rather more closely than do other groups of Bulge stars, enhancing their potential as tracers of galactic structure. The recent review by Whitelock (1990) gives more background to the subject while that by Whitelock \& Feast (1992) concentrates on the evolutionary connection between Miras and planetary nebulae. The papers by Habing and Dejonghe in these proceedings describe the OH/IR sources which are a particularly interesting subgroup of the Mira variables.

The final section of the review considers the enigmatic Bulge carbon stars. This is a topic which the organizers asked me to cover, although the carbon stars are not obviously related to the red variables. 


\section{Red Variables in the Bulge}

The early surveys of the Bulge, such as those by Gaposchkin, Oosterhoff and Plaut, used blue photographic plates and concentrated on finding RR Lyrae stars. They also noted the presence of numerous short period (mostly $\mathrm{P}<300$ day) red variables, including many Mira and semi-regular (SR) variables. This supported early ideas that the Bulge variable star population was similar to that found in globular clusters. More recent surveys using red sensitive plates, such as those by Terzan and collaborators (e.g. Terzan \& Ounnas 1988), have identified larger numbers of LPVs but are far from being complete. Lloyd Evans (1976) provided the only survey specifically aimed at finding red variables and determining their periods. He used near-infrared (I) and V plates and found 121 large amplitude red variables in the Sgr I, Sgr II and NGC 6522 Baade windows. This is the most complete survey to date for Mira variables in the Bulge, although others are now in progress. It is not however complete for the longer period, dust-enshrouded, variables with energy distributions which peak in the infrared (see e.g. Glass 1986). The IRAS satellite was particularly sensitive at detecting this kind of star as recent surveys have shown (e.g., Feast 1986, Whitelock et al. 1986, te Lintel Hekkert 1990, Whitelock et al. 1991-hereafter WFC, Blommaert 1992).

\section{Red Variables in Globular Clusters}

Studies of globular clusters have provided much of our basic understanding of red variables. The most luminous stars in globular clusters are all variable and the most luminous of the variables are the Miras (see Fig 1 of Feast \& Whitelock 1987). These all have bolometric luminosities $\mathbf{M}_{b o l}<-3.5 \mathrm{mag}$, the approximate luminosity at which the core helium-flash occurs. For this reason it is clear that they are on the asymptotic giant branch (AGB); furthermore there are no stars brighter than the Miras and we therefore presume that they are the last, reasonably long lived, phase of AGB evolution. Our current picture of the Bulge is consistent with this, although there is much greater ambiguity attached to the brightness of individual stars due to the line-of-sight depth of the Bulge.

The SR variables within a particular cluster obey a period luminosity (PL) relation, the slope of which is that predicted for an evolutionary track (Feast 1989). It thus appears that SR variables evolve into Miras. The combined Miras from all clusters containing such variables, obey a very tight PL relation (Menzies \& Whitelock 1985; Feast 1984) which is too steep to be understood as an evolutionary track. For this and other reasons the Mira PL relation is interpreted as the locus of end points of AGB evolution for stars of different initial mass (Feast \& Whitelock 1987). We might expect the SRs in the Bulge to be similar to those in globular clusters but rather little is known about these stars beyond the fact that they are numerous.

Figure 1 illustrates the PL relation for Galactic Miras with independent distances. The distances of the globular cluster Miras were calculated assuming that the magnitude of their horizontal branch, $M_{V}(H B)$, is given by: $M_{V}(H B)=$ $0.15[\mathrm{Fe} / \mathrm{H}]+0.73$ (Walker 1992), except for $\omega$ Cen where $M_{V}(H B)=0.25$ (Dickens 


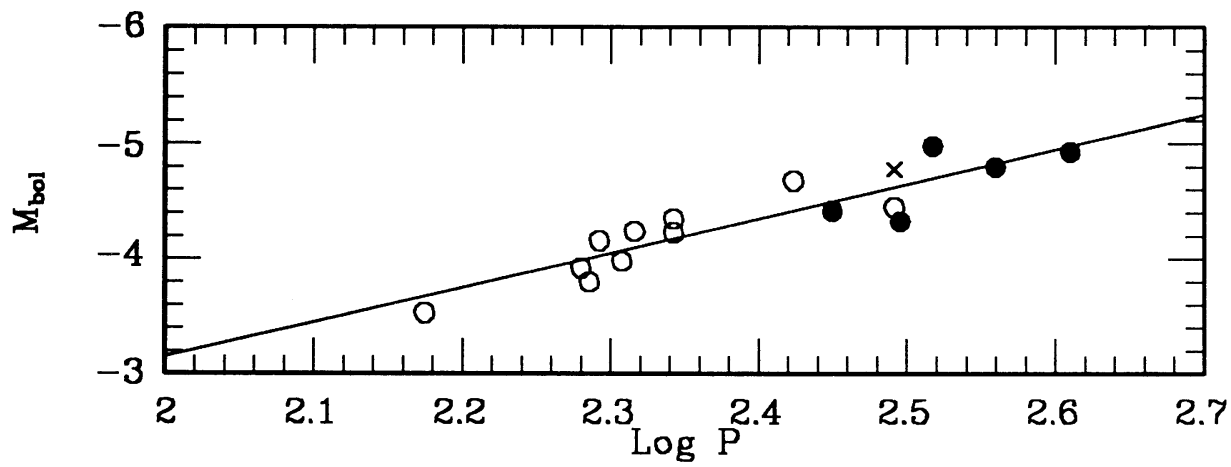

Fig. 1. The PL relation for Galactic Miras. Globular cluster photometry (open circles) is from Menzies \& Whitelock (1985) with distances derived as described in the text. The photometry for the other stars comes from Catchpole et al. (1979) and unpublished SAAO observations. Distances were derived from Robertson \& Feast (1981) for those with spectroscopic parallaxes (closed circles) and from the trigonometric parallax for $\mathrm{R}$ Leo (cross, Gatewood 1992). The line follows the LMC PL relation assuming that (m-M) $)_{0}=18.5$ mag; it is not fitted to the points.

1989) was used. The bolometric magnitudes were calculated from infrared photometry as described by Menzies \& Whitelock (1985). The line has not been fitted to the points, it is simply the PL relation derived for Miras in the LMC (Feast et al. 1989) on the assumption that the distance modulus to the LMC is 18.5. As discussed by Walker $(1992)(\mathrm{m}-\mathrm{M})_{\mathrm{o}}=18.5$ for the $\mathrm{LMC}$ is consistent with values derived from the Cepheid variables, main-sequence fitting to clusters and the ring around SN 1987A. It is clear that, within the illustrated period range, the same $\mathrm{PL}$ relation applies to Miras in the LMC, the Galactic globular clusters and the solar neighbourhood.

Within the globular clusters, Mira variables are found only in the most metalrich clusters. Some of the metal deficient clusters contain SRd variables with luminosities which suggest that they are near the top of the GB or AGB. Like Miras they show emission lines at certain phases of their variability cycle. They have earlier spectral types, lower amplitudes and shorter periods than do the Miras; differences which can be understood as a consequence of their lower metallicities. These SRd variables can be viewed as a metal-deficient analogue of the Miras. The Mira and SRd pulsation periods are a function of the metallicity of the parent cluster (Feast 1981). The longest period Mira thought to be a cluster member has a period of about 300 day and is associated with a cluster of near solar metallicity (NGC 5927). Unfortunately once we leave the clusters there is no way of determining the metallicity of a Mira. The distribution of periods among Bulge Miras covers a larger range than the clusters extending to 700 day or more. There is as yet no strong evidence that the short-period Bulge Miras differ from those found in globular clusters. The longer period objects we presume have originated from progenitors which are more metal rich than those found in the clusters, although 


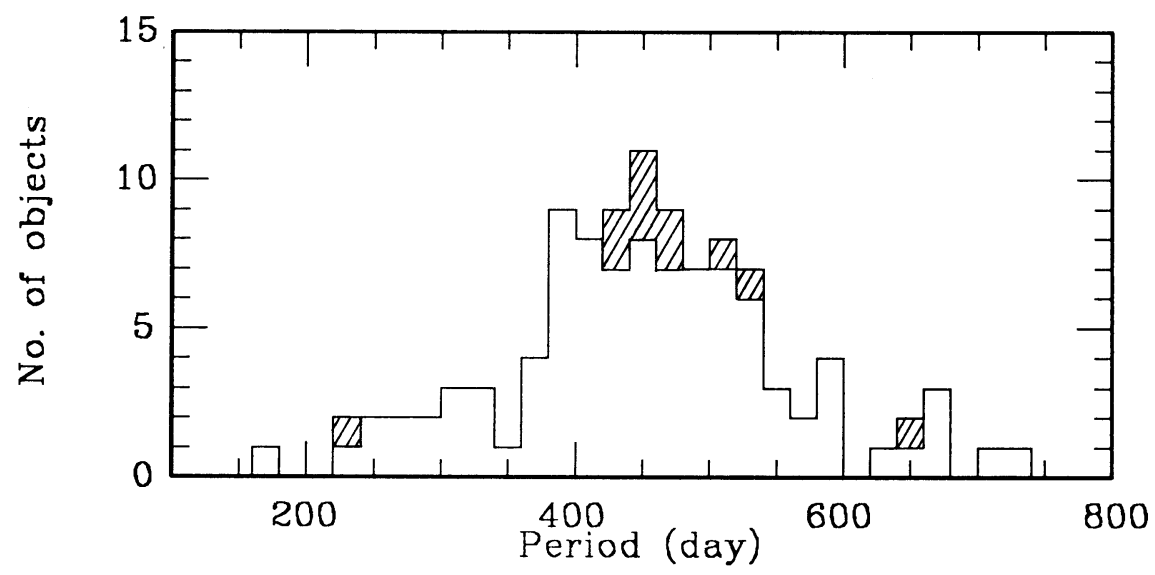

Fig. 2. Histogram of the periods of the IRAS Miras discussed by WFC. The shaded area represents those stars which are within $5.5 \mathrm{kpc}$ of the sun.

the longest period objects may originate from merged binaries (Renzini \& Greggio 1990), and are therefore not necessarily very metal rich.

\section{Mira Variables in the Galactic Bulge}

WFC published the results of a survey for Miras from two strips across the Bulge covering galactic latitudes of $b=+7^{\circ}$ to $+8^{\circ}$ and $b=-7^{\circ}$ to $-8^{\circ}$. The sources were selected from the IRAS point source catalogue (1985) as having colours indicative of high mass-loss rates. Periods were determined for 104 of the 113 Miras discovered and Fig 2 illustrates the distribution of these periods. Miras closer than $5.5 \mathrm{kpc}$ were classified as foreground to the Bulge and indicated as such on the histogram; about $13 \%$ of the IRAS Miras fell into this category. It is notable that the period distribution of these stars covers the same range as that of the Bulge Miras. It is possible that these Miras represent an extension of the Bulge population into the solar neighbourhood. They may be related to the stars discussed by Grenon (1990) which he suggests formed in the Bulge with orbits which bring them into the solar neighbourhood.

\subsection{Gradients within the Bulge}

The WFC survey covered only Miras with high mass-loss rates. It is of considerable interest to obtain a complete sample of Miras in a number of well defined regions of the Bulge in order to examine gradients as a function of position. With the intention of isolating a complete sample of Miras at $l \sim 0^{\circ}$ and $b \sim-7^{\circ} .5$, all of the IRAS sources in the 3.8 square degree area where the $-7^{\circ}$ to $-8^{\circ}$ strip overlaps the Baade/Plaut field number 3 were examined (Whitelock, Catchpole \& Marang in prep.). JHKL photometry was obtained for all the $12 \mu \mathrm{m}$ IRAS sources in this region which had not already been examined by WFC. Of the 89 IRAS sources 
observed 47 have the colours of Miras and periods were determined for 40 of these. These periods cover approximately the same range as those measured by WFC but are somewhat lower on average; very few Miras had periods less than 350 day. The mass-loss rates for these stars are discussed by Whitelock \& Feast (1992). Within the same 3.8 square degrees there were 45 Miras discovered by Plaut (1971) (see also Wesselink 1987). Most of these had periods less than 300 day. Only 4 of the IRAS Miras were in Plaut's list and 2 of these were clearly foreground stars. This lack of overlap between the Plaut and IRAS samples indicates that the sample is incomplete. The period distributions of the various samples suggests that the missing stars will be mostly in the 300 to 400 day period range.

There is considerable evidence that a metallicity gradient exists in the Bulge (e.g., van den Bergh \& Herbst 1974, Terndrup 1988, Frogel et al. 1990). The gradient is in the sense that there is a greater concentration of metal-rich stars towards the Galactic Centre, although the slope and extent of this gradient is still subject to some uncertainty. It is interesting to examine what the Miras tell us about such gradients. Unfortunately, there is, as yet, no reliable way to establish the metallicities of Miras directly. We would expect long-period Miras to have evolved either from an old metal-rich population or from any intermediate age population which may be present. Terndrup's (1988) result on the main-sequence turn-off seems to rule out a large intermediate age population. So in the first instance it seems reasonable to assume that Miras in the Bulge with periods of over $\sim 400$ day are old but probably have metallicities above the solar value. As stated above there are reasons to think that variables in the long-period tail of the period distribution may be in part the result of binary mergers and are therefore not necessarily very metal rich.

Figure 3 illustrates histograms of the period distribution for Miras in three Bulge fields at different galactic latitudes. Note that the distributions have not been normalized and that the high latitude field covers a larger area (3.9 square degrees) than do the Baade window fields (0.33 square degrees). All three histograms show a large range of periods from around 150 day to over 600 day. This is consistent with our views of a range of metallicities within the Bulge population. We also know from WFC that there are Miras with periods in the range 700-800 day present in other parts of the $7^{\circ}<|b|<8^{\circ}$ field but that they are rare.

As stated above the survey of the $b=-7^{\circ} .5$ field is probably incomplete for periods between 300 and 400 day. For this reason the only reliable way to examine differences between the distributions is to take the ratio, $\Re$, of the number of short period (200 $\leq \mathrm{P} \leq 300)$ to the number of long period $(400 \leq \mathrm{P} \leq 600)$ Miras. The following values were derived: Sgr I $\Re=1.1 \pm 0.3$; NGC $6522 \Re=1.8 \pm 0.7$; $b=-7^{\circ} .5 \Re=3.9 \pm 1.5$; where the errors are calculated assuming $\sqrt{\mathrm{N}}$ statistics. There thus appears to be a difference between the inner and outer fields in the sense that the inner field has proportionally almost four times as many long-period Miras as does the outer field. It must however be remembered that there are differences in the way that Miras were discovered in the various fields and that this conclusion may have to be modified when any systematic effects are taken into account. Note in particular that Fig 3 includes Miras which are not strictly part of the Bulge but which lie in the foreground. This will be a slightly more serious contamination of 


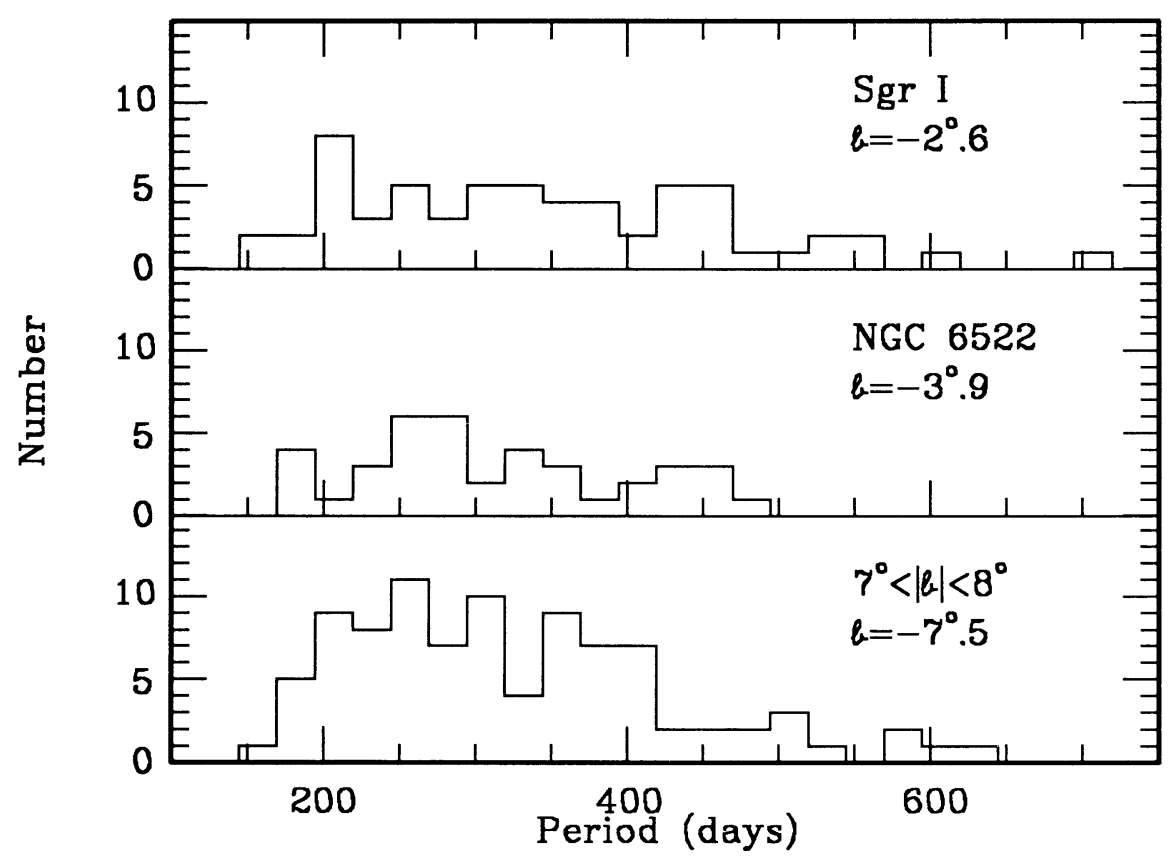

Fig. 3. The period distribution of Miras in the Bulge. The periods for the Miras are from Glass et al. (in prep.) for Sgr I, Lloyd Evans (1976) for NGC 6522 and from Whitelock et al. (in prep.) for $b=-7^{\circ}$.

the outer field than the inner ones, but correcting for this effect should not grossly affect the result. There are also Miras in the $b=-7^{\circ} .5$ field for which the periods have not been determined.

It is also interesting to note that the median of the distribution for the NGC 6522 field is at about 300 day. Rich (1988) finds that the median metallicity for K giants in the same window is at $[\mathrm{Fe} / \mathrm{H}] \sim 0$. Thus if all of the $\mathrm{K}$ stars evolve into Miras and if stars of different metallicity spend proportionally the same time as $\mathrm{K}$ giants and as Miras, then a 300 day Bulge Mira must have $[\mathrm{Fe} / \mathrm{H}] \sim 0$. This would be consistent with what is known from Miras in globular clusters. Note however that Miras are absent from the metal deficient clusters.

Blanco (1988) has pointed out differences in the radial gradient of early- $\mathrm{M}$ and of late-M stars. Figure 4 compares the radial gradient of the Miras with that of the late-M stars (M7 and later from Blanco 1988). The M stars have been corrected for foreground stars, the Miras have not. As discussed above, all three fields surveyed for Miras are probably incomplete, but the outermost field $\left(b=-7^{\circ} .5\right)$ is likely to be less complete than the other two. The two solid lines on Fig 4 are exponentials with scale heights of $2^{\circ} .7$, the value determined by Kent et al. (1991) for the $2.4 \mu \mathrm{m}$ 


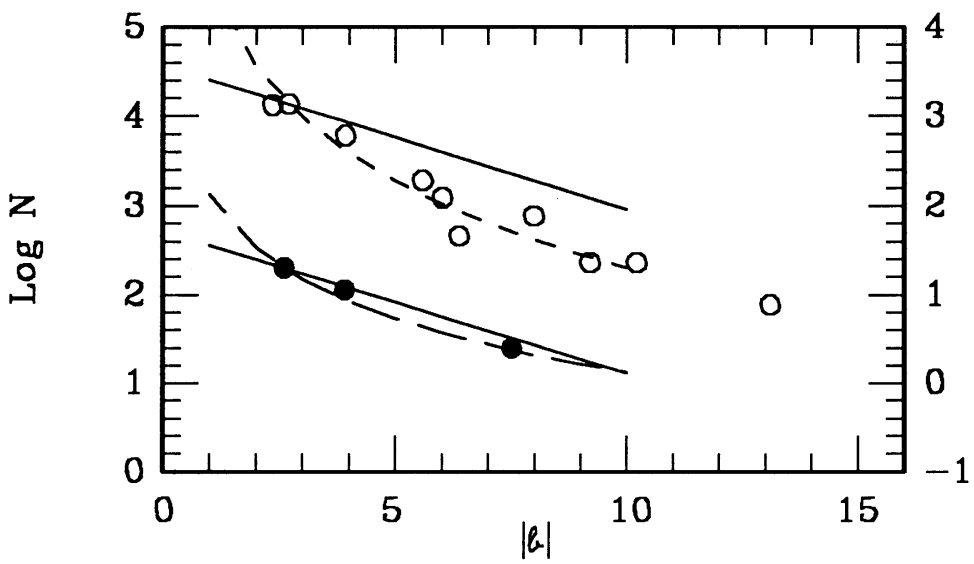

Fig. 4. The number of Miras (closed circles, left axis) compared to the number of late-M stars (open circles, right axis) per square degree as a function of galactic latitude, $b$. The solid lines are drawn at the slope of the model from Kent et al. (1991) for the logarithmic $2.4 \mu \mathrm{m}$ surface luminosity. The broken lines are the power-laws discussed in the text. All of the lines are normalized to the measurements for the Sgr I window.

radial surface brightness within $10^{\circ}$ of the Galactic Center. They are normalized to the number of Miras and $\mathrm{M}$ stars at $|b| \sim 2^{\circ} .7$, respectively. This comparison of surface brightness and surface number density implies an assumption that the luminosity function remains constant over the range of latitude considered. Such an assumption, although not strictly justified, may be a reasonable first approximation. The difference between the distribution of $\mathrm{M}$ stars and that of the $2.4 \mu \mathrm{m}$ surface brightness has been been commented on by Tyson (1992). Figure 4 illustrates two points of particular interest. First, the trend shown by the Miras is indicative of the behaviour of the general Bulge luminosity, and it seems likely that the same will be true in extragalactic bulges. Secondly, the late-M stars have a steeper radial gradient than do the Miras.

The radial gradient in volume number density, $N$, is sometimes expressed as a power law of the distance from the Galactic Centre, $R$, so that $N \propto R^{-\alpha}$. The equivalent expression for the surface number density, $\rho$, as illustrated in Fig 4 , is then $\rho \propto R^{1-\alpha}$ over the range of latitude under consideration. For late $\mathrm{M}$ stars $\alpha \sim 4.2$ (Blanco \& Terndrup 1989) as is illustrated by the line of short dashes in Fig 4. A value of $\alpha \sim 3$ provides a good fit to the Mira densities as is shown by the line of long dashes. Note however that a preliminary analysis of the line-of-sight distribution of the Miras in Sgr I requires $\alpha \sim 2$ (Whitelock \& Feast 1992 Fig 2 and Glass et al. in prep.). The difference between the radial gradient discussed above and the line-of-sight distribution may be a consequence of the triaxial nature of the Bulge (see $§ 4.2$ ).

The difference in the radial distribution of Miras and late-M stars is puzzling, since it is generally assumed that the $\mathrm{M}$ stars, whether they are on the giant branch 
(GB) or the AGB, evolve into Miras. It also appears, from the comparison with the $2.4 \mu \mathrm{m}$ surface luminosity, that the behaviour of the late-M stars is not indicative of the luminous red, presumably $\mathrm{K}$ and early-M, Bulge stars Possibly the very late-M stars (i.e. the most metal rich ones) take some alternative evolutionary track and do not become AGB stars in the normal way. The late evolution of metal rich stars has been discussed in detail by Greggio \& Renzini (1990) (see also Horch et al. $1992)$ in an attempt to explain the ultraviolet excess observed in super-metal-rich elliptical galaxies. It appears that under certain conditions metal rich stars may not undergo AGB evolution. It is not yet clear if we should expect this kind of scenario to apply to stars in the Galactic Bulge. Two alternative explanations of the different distributions are that the Mira descendants of metal-rich $\mathbf{M}$ giants have much shorter lifetimes than their globular cluster counterparts or that the late-M giants evolve into long-period Miras and that these have a much steeper density gradient than do short period ones. Better statistics for the Miras are necessary before progress can be made with this aspect of the problem.

In looking at the broad question of density gradients in the Bulge it is interesting to note that the RR Lyrae variables show an even steeper gradient than the lateM stars between $|b| \sim 3^{\circ} .9$ and $|b| \sim 8^{\circ}$ as can be seen from Fig 1 in Feast et al. (1992). Note that the point representing the RR Lyraes at $|b| \sim 3^{\circ} .9$ on this diagram has not been corrected for completeness and the true slope is therefore steeper than it appears. This is in contrast to the gradient of RR Lyraes at higher latitudes which is shallower than that of the $M$ stars. In addition it is now known (Walker \& Terndrup 1991, Wesselink 1987) that the RR Lyrae variables at $b \sim 3^{\circ} .9$ are more metal rich than those at higher latitudes. Therefore the metal-rich $\mathrm{RR}$ Lyraes (those with $[\mathrm{Fe} / \mathrm{H}] \sim-1$ ) must have an extremely steep density gradient. This suggests that the interpretation of gradients in the Bulge may not be entirely trivial.

\subsection{Structure of the Bulge}

Mira variables have a considerable, but largely unrealized, potential for the study of Galactic structure. This is because the distances to individual stars can be determined by use of the PL or PLC relations. In practice of course determining periods and mean luminosities for large numbers of variables is a time consuming activity. Whitelock \& Catchpole (1992) (see also Whitelock 1992) demonstrated the usefulness of this approach in examining the structure of the Bulge. They looked at the distribution of distance moduli for IRAS Miras from WFC, separating them into two groups depending on whether they were in the first $(l>0)$ or fourth $(l<0)$ Galactic quadrant. The distributions are illustrated in Fig 5a together with the best fitting model of a triaxial ellipsoidal Bulge. It is clear from this that the peak of the distribution at negative longitudes is further from us than the peak on the other side. Figure $5 \mathrm{~b}$ shows the longitude distribution for the same sources and model. The model illustrated is of the form:

$$
N \propto \exp \left[-\left(\frac{x}{x_{\circ}}\right)^{2}-\left(\frac{y}{y_{\circ}}\right)^{2}-\left(\frac{z}{z_{\mathrm{o}}}\right)^{2}\right]^{1 / 2}
$$



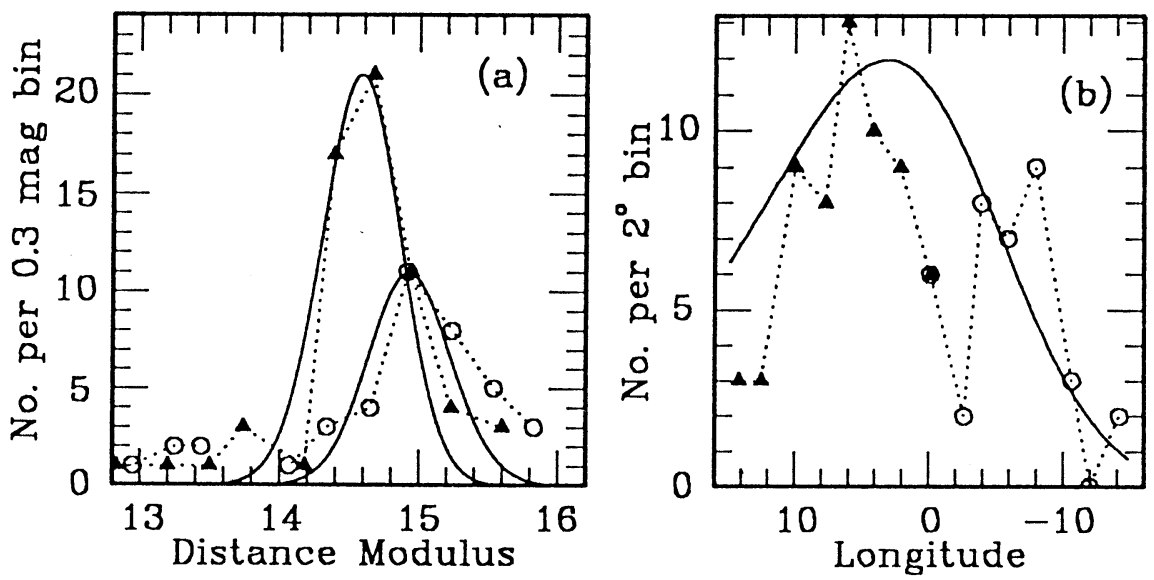

Fig. 5. (a) The distribution of IRAS Miras in distance modulus. The open circles and closed triangles represent those with negative and positive longitudes respectively. (b) The latitude distribution of the same sources. The solid lines represent one of the best fitting models, as discussed in the text.

and the best fitting scale lengths are $x_{0}=700 \mathrm{pc}, y_{0}=z_{0}=175 \mathrm{pc}$ and the $x$ axis is inclined at an angle $\theta=45^{\circ}$ to the line of sight. A rather large distance to the Galactic Centre, $R_{0}=9.1 \mathrm{kpc}$, is required for this fit, because the distances to the Miras were obtained from the PL rather than the PLC relation, the latter not being applicable to Miras with thick dust shells. Because rather few Miras were used to define this model and because of possible systematic biases in the IRAS data-base, the exact parameters of the fit should not be over interpreted. In particular, if the differences in the distributions of Miras along the minor axis of the Bulge and in the line of sight, which were discussed in $\$ 4.1$, are a consequence of Bulge triaxiality then the angle $\theta$ must be less than $45^{\circ}$ (note that Binney et al. (1991) deduce $\theta=16 \pm 2^{\circ}$ on dynamical grounds). The result does however illustrate the potential of Mira variables as detailed probes of structure within the Bulge and suggests that a detailed study of a large number of stars could be very rewarding. The derived asymmetry for the Bulge is at least qualitatively consistent with other evidence for triaxiality as summarized by de Zeeuw and by Gerhard in these proceedings. Menzies (1990) measured radial velocities for a number of the visually brighter Miras from WFC. His results show the expected high velocity dispersion but also clearly illustrate the rotation of the Bulge.

\subsection{The Period-Luminosity-Colour Relation}

A preliminary analysis of multi-phase IR observations of Miras in the Sgr I window $\left(l=1^{\circ} .4, b=-2^{\circ} .6\right)$ suggests that these stars diverge somewhat from the LMC PL and period-colour (PC) relations (Glass, Whitelock, Feast \& Catchpole in prep.). A comparison of the $(\mathrm{J}-\mathrm{K})$ colours of the Bulge and LMC Miras is shown in Fig 
1 of Whitelock \& Feast (1992). It is clear from this that the longer period Bulge sources have redder colours than would be predicted from the LMC PC relation. Note that the Miras with circumstellar reddening $\left((\mathrm{J}-\mathrm{K})_{\circ} \gtrsim 4.0\right)$ were omitted from consideration in the first instance as their photospheric colours cannot easily be deduced.

The Galactic Centre is sufficiently close to us that we observe a finite spread in the line of sight for the distance moduli of Bulge stars. This spread means that we cannot assume that any given Bulge star is at the distance of the centre. In order to compare the luminosities of Bulge Miras with those in the LMC the following procedure was adopted. The LMC PL relations, expressed in terms of bolometric and $\mathrm{K}(2.2 \mu \mathrm{m})$ magnitude (Feast et al. 1989), were assumed to apply to the Bulge Miras and used to derive individual distance moduli. The resulting distributions of distance moduli can be fitted with models for the distribution of stars in the Bulge from which the distance to the centre can be derived. The result is $R_{\circ}=9.1 \mathrm{kpc}$ from the bolometric PL and $R_{\circ}=8.6 \mathrm{kpc}$ from the $\mathrm{K} \mathrm{PL}$ (note that although the absolute error on these distances is at least $\pm 0.5 \mathrm{kpc}$, the relative error is only $\pm 0.1 \mathrm{kpc}$ ). These values not only disagree, they are significantly further than the currently accepted distance of $R_{\circ}=7.8 \pm 0.8 \mathrm{kpc}$ (Feast 1987). This strongly suggests that the Bulge Miras are fainter than we would expect from the LMC PL relations. However, if the distance moduli are derived via the LMC PLC relations then both of the resulting distributions can be fitted by models with $R_{\circ}=8.2 \mathrm{kpc}$. Further work is in progress but this seems to confirm the existence of the PLC relation and imply that the Bulge Miras, or at least the longer period ones, occupy a different part of the instability strip from the LMC Miras, probably due to metallicity effects (see Wood et al. 1991).

The implications of this are quite significant (see also §4.4). It is clear that in principle the PLC relation should be used in preference to the PL relation for determining distances to Miras. However, in practice the PL relation seems quite adequate for most environments (see Fig 1). It is probable that difficulties with the PL relation will only manifest themselves in very metal-rich environments. The distances to the long-period ( $\mathrm{P}>600$ day) Galactic $\mathrm{OH} / \mathrm{IR}$ stars are rather poorly known (van Langevelde et al. 1990) but on average their luminosities fall below an extrapolation of the LMC PL relation (WFC), as do those of the Bulge Miras. This is in contrast to the LMC OH/IR sources (Wood et al. 1991) which lie on an extrapolation of the Mira PL relation. It is possible therefore that some of the Galactic OH/IR sources may be less luminous than their LMC counterparts. In view of this the procedure used by WFC to derive a Mira PL relation, by combining data on LMC Miras and Galactic OH/IR sources (their equation 2), may not be strictly valid. However, much more accurate distances are required for the Galactic OH/IR sources before firm conclusions can be drawn on this point.

Blommaert et al. (1992) have made a detailed study of a few of the OH/IR stars within 30 arcmin of the Galactic Centre, and pointed out that they are fainter than would be predicted from the LMC PL relation (see their Fig 3). These are particularly valuable observations because the concentration of stars near the centre is such that it is possible to assume that almost all the sources are at the distance of the centre. There are distinct difficulties in doing photometry of these stars due 


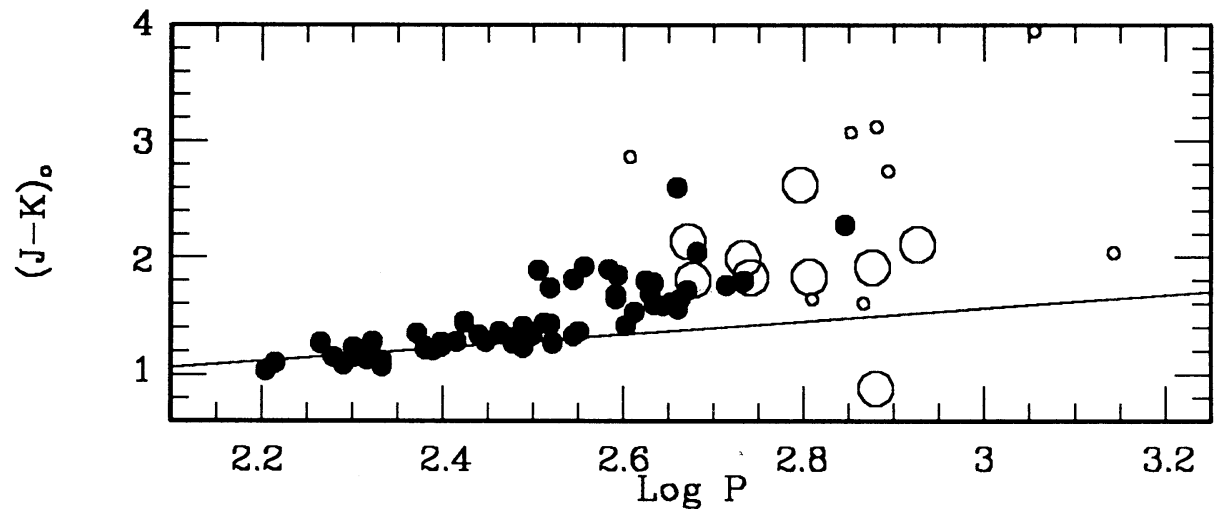

Fig. 6. A PC diagram comparing the observed reddening-corrected colours of the Sgr I Miras (closed circles, Glass et al. in prep.) with those calculated using the LMC PLC relation for the Galactic Centre OH/IR sources (large open circles for those with accurate periods and luminosities, small circles for those with uncertain periods and/or luminosities) from Blommaert et al. (1992). The line is the PC relation for LMC Miras from Feast et al. (1989).

to the very crowded nature of the fields and to the high, but uncertain, interstellar extinction corrections. Unfortunately it is not possible to apply the PLC relations directly to these sources as they have thick dust shells so that their (J-K) colours are strongly influenced by circumstellar extinction. However, we can reverse the problem and assume that they are at the distance of the Galactic Centre $\left(R_{\circ}=8.0\right.$ $\mathrm{kpc}$ ) and use the PLC relation to calculate their photospheric (J-K). This has been done and in Fig 6 the result is compared to the measured colours of the Sgr I Miras. Those $\mathrm{OH} / \mathrm{IR}$ sources with accurate luminosities and periods follow the trend established by the slightly shorter period Sgr I Miras (with one exception which might be closer than the centre, or in a different evolutionary state from the others). This suggests that these $\mathrm{OH} / \mathrm{IR}$ sources might also follow the same PLC relation as the LMC Miras.

\subsection{Numbers and Luminosities of Miras in the Bulge}

It is possible to combine the data from the WFC survey with the estimate of the total number of Miras in the 3.8 square degree overlap with the Baade/Plaut field (described at the beginning of $\S 4.1$ ) to calculate the total number of Miras in the Bulge, bearing in mind that the survey was probably incomplete for Miras with periods in the 300 to 400 day range. There were 95 Miras in 3.8 square degrees seven of which were in the WFC survey which had a total of 113 high-mass-loss Miras from two one-degree wide strips across the Bulge. Therefore these two strips will contain a total of approximately $(113 / 7) \times 95(-15 \%$ foreground $)=1300$ Miras of all kinds. Assuming that the density distribution of Miras in the Bulge falls off as 
$\mathrm{R}^{-2}$ then the total number of Miras within a radius of $2 \mathrm{kpc}$ from the centre must be at least $2 \times 10^{4}$. If the Mira population is actually more centrally concentrated than these assumptions imply then the total number will be larger.

The lifetime of a Bulge Mira may be estimated via Renzini \& Buzzoni's (1986) fuel consumption theorem, $N_{j}=B(t) L_{T} t_{j}$. This relates the number of objects, $N_{j}$, in a specific evolutionary phase with the duration of that phase, $t_{j}$, via the total luminosity of the population, $L_{T}$, and the stellar death rate per unit luminosity, $B(t)$. Assuming $B(t) \sim 2 \times 10^{-11} L_{\odot} y r^{-1}$ and $L_{T} \sim 10^{10} L_{\odot}$ (Renzini \& Greggio $1990)$ we find a Mira lifetime of at least $10^{5} \mathrm{yr}$. This can be compared with other estimates: Renzini \& Greggio found $2.5 \times 10^{5} \mathrm{yr}$ for the globular cluster Miras using the same expression - these are short period objects ( $\mathrm{P}<300$ day); Jura \& Kleinmann (1992) have recently estimated $2 \times 10^{5} \mathrm{yr}$ for Miras with intermediate periods (300-400 day) in the solar neighbourhood. Although the Bulge Miras show a large range of periods, from 100 to perhaps 800 day, the bulk of them have periods less than 400 day.

There is a good deal of uncertainty surrounding the luminosity of the brightest stars in the Galactic and other bulges (see e.g. the contributions to this meeting by Rich and by Renzini). The most luminous stars are assumed to be representative of the last significant stage of star formation, although it is not entirely clear how chemical composition affects the chronology. Provided an old or intermediate age population is sufficiently large that it includes stars in the short lived Mira phase then these stars will be the most luminous of that population. In view of the above findings it is clear that luminosities of Bulge Miras which were determined via the PL relation will have been overestimated. Extrapolating from WFC and more recent work in the $b=-7^{\circ} .5$ field it is possible to estimate that the Bulge will contain very roughly 100 stars with luminosities greater than $\mathrm{M}_{b o l}=-5.0 \mathrm{mag}$. As already stated these may be the result of binary mergers rather than the evolution of stars with $M_{i}>1.2 M_{\odot}$. However this estimate does not take into account the luminous stars which have a highly flattened distribution around the Galactic Centre (Catchpole et al. 1990, Feast 1989 and Whitford these proceedings) and must therefore be regarded as a lower limit. Evidence for luminous stars near the Galactic Centre and in the bulges of other galaxies is discussed in Rich's contribution to these proceedings.

Whitelock \& Feast (1992) compare the relative numbers and ages of Miras and planetary nebulae in the Bulge. They conclude that Miras outnumber planetary nebulae by about 10 to 1 , although there is a high level of uncertainty in the assumptions made about both types of object. They also conclude that there are no compelling reasons to doubt that most, if not all, Miras evolve into planetary nebulae. However, the large number of planetary nebulae that seem to have evolved from binary systems (e.g. Livio 1992) may cast doubt on the idea that most planetary nebulae have evolved through a Mira phase.

\section{The Bulge Carbon-Stars}

Azzopardi et al. (1991) have published coordinates and finding charts for the carbon stars in the Bulge. Their astrophysical properties have been discussed by Tyson 
\& Rich (1991) and Westerlund et al. (1991) and, except where noted, it is upon these two papers that the following summary is based. One of the most notable characteristics of these Bulge carbon stars is their low luminosity $\left(\mathrm{M}_{b o l} \gtrsim-2.5\right.$ $\mathrm{mag})$. They therefore cannot be in a similar evolutionary state to the stars discussed above. They could only be near the end of their AGB evolution if they are going to evolve off the AGB prematurely, to become post early-AGB stars, but according to Greggio \& Renzini (1990) such stars will be nitrogen-rich and carbon-deficient. They are certainly not near the top of any normal AGB, where the Miras are, nor can they be on the thermal pulsing part of the AGB. That being the case their carbon enrichment cannot result from dredge-up following a thermal pulse, as is thought to occur for the luminous carbon stars found in the Magellanic Clouds and elsewhere: The Bulge carbon stars are very rare, being outnumbered several hundred to one by $\mathrm{M}$ stars, so they must represent either a short-lived phenomenon or one which occurs only in a small fraction of the population, or both. These stars are presumed to be metal-rich because of their strong absorbtion features, in particular $\mathrm{Na} \mathrm{D}$ and $\mathrm{CN}$ bands.

Azzopardi et al. (1988) suggested the Bulge carbon stars might have become enriched in carbon through binary mass-transfer, presumably by analogy with the barium and $\mathrm{CH}$ stars. However, the discovery that many of the Bulge carbon stars have enhanced ${ }^{13} \mathrm{C}$ but no s-process enhancement led both Tyson \& Rich and Westerlund $e t$ al. to reject the binary hypothesis and to suggest instead that the Bulge stars may be closely related to the R-type carbon stars. These are not generally thought to be binaries and their carbon enrichment is as yet unexplained although much of the literature favours a suggestion made by Dominy (1984) that the enrichment could be associated with mixing during the core-He flash.

It seems to me that recent work on dwarf carbon stars is highly relevant to this problem. There are four known dwarf carbon stars (Green et al. 1991). Limited spectroscopic studies have shown that at least two and possibly three of these have enhanced ${ }^{13} \mathrm{C}$ without any obvious overabundance of the s-process elements (Lloyd Evans 1992 \& refs therein). If these stars are indeed dwarfs then any explanation for the anomalous abundances involving the core-He flash is out of the question and binary interaction would seem to offer the only viable solution. It therefore seems reasonable to suggest that both the dwarf- and the Bulge carbon stars must originate from binary interactions. More work is required to confirm the apparent similarity of the abundance peculiarities found in the dwarfs and the Bulge carbon stars.

\section{Acknowledgements}

I am grateful to Michael Feast and John Menzies for helpful discussion. I would also like to thank Robin Catchpole, Michael Feast and Ian Glass for permission to quote work in preparation.

\section{References}

Azzopardi, M., Lequeux, J. \& Rebeirot, E.: 1988, Astron. Astrophys., 202, L27 
Azzopardi, M., Lequeux, J., Rebeirot, E. \& Westerlund, B. E.: 1991, Astron. Astrophys. Suppl., 88,265

Binney, J., Gerhard, O. E., Stark, A. A., Bally, J. \& Uchida, K. I.: 1991, Mon. Not. R. astr. Soc., 252, 210

Blanco, V. M.: 1988, A stronom. J., 95, 1400

Blanco, V. M. \& Terndrup, D. M.: 1989, Astronom. J., 98, 843

Blommaert, J. A. D. L.: 1992, PhD thesis, Rijksuniversiteit te Leiden

Blommaert, J. A. D. L., van Langevelde, H. J., Habing, H. J., van der Veen, W. E. C. J. \& Epchtein, N.: 1992, in Variable Stars and Galaxies, ASP conf. ser. 30, ed., B. Warner, in press

Catchpole, R. M., Robertson, B. S. C., Lloyd Evans, T. H. H., Feast, M. W., Glass, I. S. \& Carter, B. S.: 1979, $S A A O$ circ., 1, 61

Catchpole, R. M., Whitelock, P. A. \& Glass, I. S.: 1990, Mon. Not. R. astr. Soc., 247, 479

Dickens, R. J.: 1989, in The Use of Pulsating Stars in Fundamental Problems of Astronomy, IAU Coll 111, ed., E. G. Schmidt, Cambridge Univ. Press, p. 141

Dominy, J. F.: 1984, A strophys. J. Suppl., 55, 27

Feast, M. W.: 1981, in Physical Processes in Red Giants, eds., I. Iben \& A. Renzini, Reidel, p. 193

Feast, M. W.: 1984, Mon. Not. R. astr. Soc., 211, 51P

Feast, M. W.: 1986, in Light on Dark Matter, ed., F. P. Israel, Reidel, p. 339

Feast, M. W.: 1987, in The Galaxy, eds., G. Gilmore \& B. Carswell, Reidel, p. 1

Feast, M. W.: 1989, in The Use of Pulsating Stars in Fundamental Problems of Astronomy, IAU Coll 111, ed., E. G. Schmidt, Cambridge Univ. Press, p. 205

Feast, M. W. \& Whitelock, P. A.: 1987, in Late Stages of Stellar Evolution, eds., S. Kwok \& S. R. Pottasch, Reidel, p. 33

Feast, M. W., Whitelock, P. A. \& Sharples, R.: 1992, in The Stellar Populations of Galaxies, IAU Sym. 149, eds., B. Barbuy \& A. Renzini, Kluwer, p. 77

Feast, M. W., Glass, I. S., Whitelock, P. A. \& Catchpole, R. M.: 1989, Mon. Not. R. astr. Soc., 241, 375

Frogel, J. A., Terndrup, D., Blanco, V. M. \& Whitford, A. E.: 1990, A strophys. J., 353, 494

Gatewood, G.: 1992, Publ. Astronom. Soc. Pacif., 104, 23

Glass, I. S.: 1986, Mon. Not. R. astr. Soc., 221, 879

Green, P. J., Margon, B. \& MacConnell, D. J.: 1991, A strophys. J., 380, L31

Greggio, L. \& Renzini, A.: 1990, A strophys. J., 364, 35

Grenon, M.: 1990, in Bulges of Galaxies, ESO-CTIO Workshop, eds., B. J. Jarvis \& D. M. Terndrup, ESO, p. 143

Horch, E., Demarque, P. \& Pinsonneault, M.: 1992, A strophys. J., 388, L53

IRAS Point Source Catalogue: 1985, US Government Publication Office

Jura, M. \& Kleinmann, S G.: 1992, A strophys. J. Suppl., 79, 105

Kent, S. M., Dame, T. M. \& Fazio, G.: 1991, A strophys. J., 378, 131

Livio, M.: 1992, in Planetary Nebulae, IAU Sym. 155, eds., R. Weinberger \& A. Acker, Kluwer, in press

Lloyd Evans, T.: 1976, Mon. Not. R. astr. Soc., 174, 169

Lloyd Evans, T.: 1992, Mon. Not. R. astr. Soc., submitted

Menzies, J. W.: 1990, in Bulges of Galaxies, ESO-CTIO Workshop, eds., B. J. Jarvis \& D. M. Terndrup, ESO, p. 115

Menzies, J. W. \& Whitelock, P. A.: 1985, Mon. Not. R. astr. Soc., 212, 783

Plaut, L.: 1971, Astron. Astrophys. Suppl., 4, 75

Renzini, A. \& Buzzoni, A.: 1986, in Spectral Evolution of Galaxies, eds., C. Chiosi \& A. Renzini, Reidel, p. 195

Renzini, A. \& Greggio, L.: 1990, in Bulges of Galaxies, ESO-CTIO Workshop, eds., B. J. Jarvis \& D. M. Terndrup, ESO, p. 47

Rich, R. M.: 1988, Astronom. J., 95, 828

Robertson, B. S. C. \& Feast, M. W.: 1981, Mon. Not. R. astr. Soc., 196, 111

te Lintel Hekkert, P.: 1990, PhD thesis, Rijksuniversiteit te Leiden

Terndrup, D. M.: 1988, A stronom. J., 96, 884

Terzan, A. \& Ounnas, Ch.: 1988, Astron. Astrophys. Suppl., 76, 205

Tyson, N. D.: 1992, in Variable Stars and Galaxies, ASP conf. ser. 30, ed., B. Warner, in press

Tyson, N. D. \& Rich, R. M.: 1991, Astrophys. J., 367, 547 
van den Bergh, S. \& Herbst, E.: 1974, Astronom. J., 79, 603

van Langevelde, H. J., van der Heiden, R., van Schooneveld, C.: 1990, Astron. Astrophys., 239, 193

Walker, A. R.: 1992, A strophys. J., 390, L81

Walker, A. R, \& Terndrup, D. M.: 1991, A strophys. J., 378, 119.

Wesselink, Th.: 1987, PhD thesis, Nijmegen

Westerlund, B. E., Lequeux, J., Azzopardi, M. \& Rebeirot, E.: 1991, A stron. Astrophys., 244, 367

Whitelock, P. A.: 1990, in Confrontation Between Stellar Pulsation and Evolution, eds., C. Cacciari \& G. Clementini, ASP Conf. Ser. 11, p. 365

Whitelock, P. A.: 1992, in Variable Stars and Galaxies, ASP conf. ser. 30, ed., B. Warner, in press

Whitelock, P. A. \& Catchpole, R. M.: 1992, in The Center, Bulge and Disk of the Milky Way, Ed L. Blitz, Kluwer, in press

Whitelock, P. A. \& Feast, P. A.: 1992, in Planetary Nebulae, IAU Sym. 155, eds., R. Weinberger \& A. Acker, Kluwer, in press

Whitelock, P. A., Feast, M. W. \& Catchpole, R. M.: 1986, Mon. Not. R. astr. Soc., 221, 1

Whitelock, P. A., Feast, M. W. \& Catchpole, R. M.: 1991, Mon. Not. R. astr. Soc., 248, 276, (WFC)

Wood, P. R., Moore, G. K. G. \& Hughes, S. M. G.: 1991, in The Magellanic Clouds, IAU Sym. 148, ed(s)., R. Haynes \& D. Milne, Kluwer, 259

\section{DISCUSSION}

Terndrup: With reference to the RR-Lyraes, Walker and I have indeed shown that the RR-Lyraes are formed only by the most extreme metal poor tail of the $\mathrm{K}$ giants. Have you looked into whether the presence of somewhat younger stars, which would also produce more RR-Lyraes in the instability strip, account for that steeper gradient in the RR-Lyra density distribution.

Whitelock: No I haven't looked into that at all. But obviously you have to look at the total picture. If you need more massive stars to explain the RR-Lyraes, then you will, presumably, end up with more massive stars becoming M-giants and Miras.

Blommaert: The Miras of 200 to 300 days period, in the Palomar-Groningen field number 3 have, I believe, mass losses of $10^{-6} M_{\odot} / \mathrm{yr}$ and the life time would be around $10^{5} \mathrm{yr}$ you say, so that would mean that they lose $10^{-1} M_{\odot)}$ in total?

Whitelock: I don't think there is any reason to suspect that the mass loss remains constant through the lifetime of a Mira. I think that there is every reason to believe that the mass-loss rate must increase during the Mira evolution. That is consistent with the fact that you find a very large range in mass loss at a given period for the Miras.

Blommaert: But these IRAS sources can be interpreted as an evolutionary stage after the Miras, because they have larger mass losses and higher mass loss rates.

Whitelock: We do have objects in different evolutionary stages and it is true that the ones in the most advanced evolutionary stage you expect to have the highest 
mass-loss rate. But this is complicated by the fact that they are thermally pulsing (i.e. undergoing helium shell flashes) and the mass-loss rate will fluctuate with the pulses. But then, we've also got a range of initial masses and the evidence, as far as the kinematics of the solar neighborhood Miras are concerned, is that Miras of different period must have different initial masses because they have quite different kinematics. Although we are probably not dealing with a large range of initial masses in the Bulge, perhaps just a few tenths of a solar mass.

Tyson: If you do appeal to binarity to explain the bulge carbon stars, then you change the focus of the problem, because you then upset what I had always interpreted to be very well understood evolutionary behavior of the secondary star that would be transferring matter.

Whitelock: I won't argue with that statement, but we also have to explain these dwarf carbon stars. They seem to have the same characteristics and unless our complete understanding of stellar evolution is wrong, then you can't get dwarf carbon stärs unless they are in binary systems.

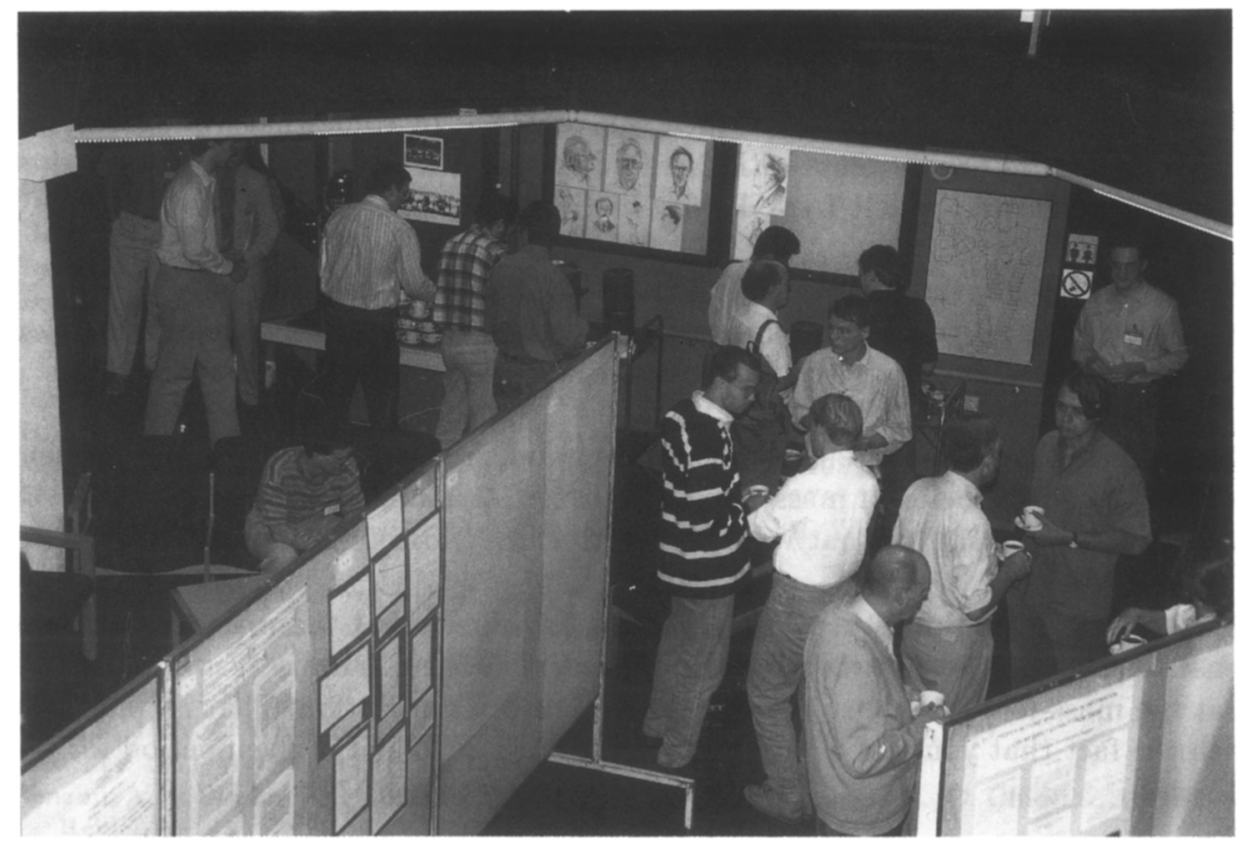

During a coffee break 


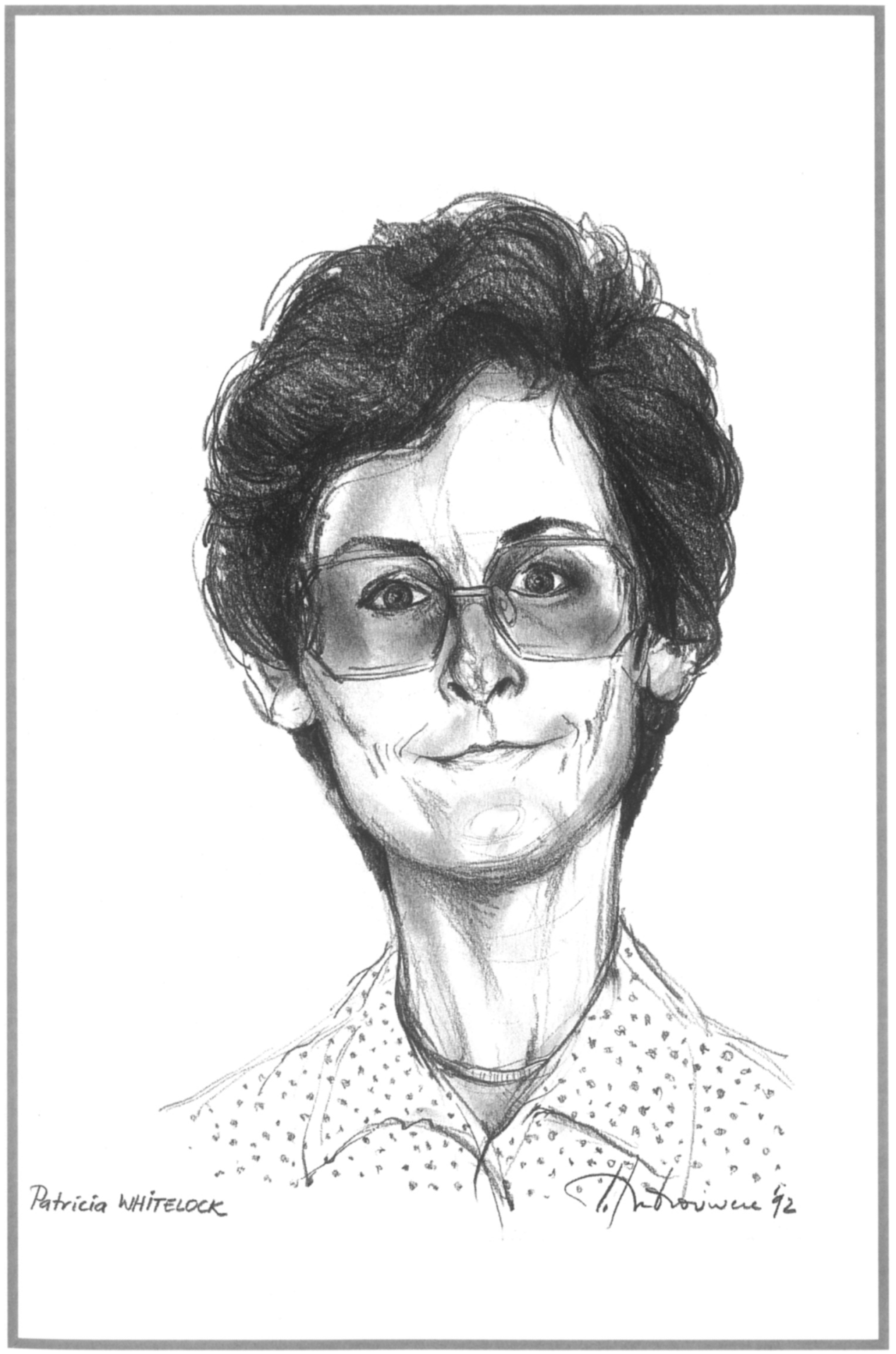




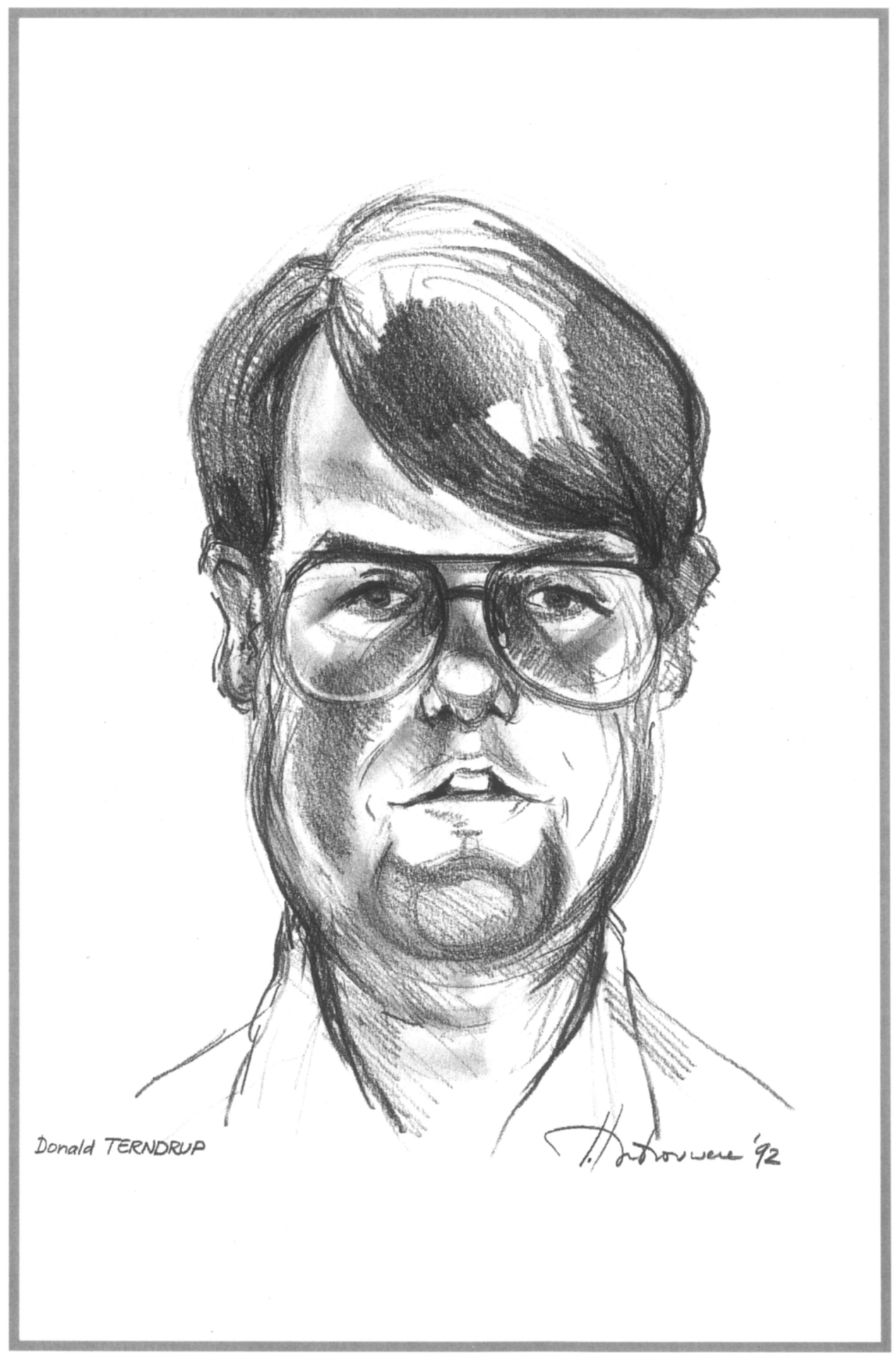

